\title{
Loop calculations for models of graphene
}

\author{
J.A. Gracey* \\ Theoretical Physics Division, Department of Mathematical Sciences, University of Liverpool, \\ P.O. Box 147, Liverpool, L69 3BX, United Kingdom \\ E-mail: gracey@liverpool.ac.uk
}

\begin{abstract}
The various Gross-Neveu classes of quantum field theories are of interest in the study of a particular phase transition in graphene. We review recent activity in the determination of several critical exponents in the chiral Heisenberg Gross-Neveu universality class using the critical point large $N$ technique which includes the use of the large $N$ conformal bootstrap method.
\end{abstract}

Loops and Legs in Quantum Field Theory (LL2018)

29 April 2018 - 04 May 2018

St. Goar, Germany

${ }^{*}$ Speaker. 


\section{Introduction}

The Gross-Neveu model, [1], has been of interest in recent years due to its connection with a phase transition in graphene. This is a one atom thick layer of carbon atoms arranged in a honeycomb lattice. In particular when the carbon sheet is deformed or stretched its electrical conductivity properties can change from a conductor to a Mott-insulating phase, [2, 3]. The critical dynamics of this phase transition is believed to be described by the chiral Heisenberg Gross-Neveu (cHGN) model, $[4,5,6,7,8]$. As the fundamental interaction of this and the other Gross-Neveu field theories is a quartic fermion self-interaction there is also a connection with the Standard Model of particle physics. At the Wilson-Fisher fixed point the ultraviolet completion of the Gross-Neveu model in four dimensions is the Gross-Neveu-Yukawa theory (GNY), [9]. In this formulation the auxiliary scalar field of the two dimensional theory is transformed into a propagating fundamental field with a quartic scalar interaction in addition to the Yukawa one. Structurally the basic Lagrangian is similar to the Standard Model. Moreover prior to the discovery of the Higgs particle such Gross-Neveu-Yukawa models were used to explore the idea that the Higgs particle was a composite of fermions. For example, see [10].

The current interest in the application to graphene centres on the determination of the critical exponents governing the phase transition. In general these can be computed for the physically relevant case of three dimensions by using techniques such as the $\varepsilon$ expansion, Functional Renormalization Group (FRG), Monte Carlo methods or the large $N$ expansion. While the Ising Gross-Neveu model has been extensively examined in perturbation theory as well as the other techniques to very high precision with in general very solid agreement across all methods, the status of the more interesting chiral Heisenberg Gross-Neveu universality class is not yet at the same level. For instance, the Functional Renormalization Group study of [8] is primarily the first reliable field theory examination of the exponents $\eta, \eta_{\phi}$ and $v$. Indeed Figures 1, 2 and 3 of [8] reflect their status. However since that article there has been significant work in the four dimensional ultraviolet completion at four loops, $[11,12,13]$. Also the same critical exponents have been determined to three orders in the large $N$ expansion, [14]. We will therefore review recent progress of the latter in this area.

\section{Background}

To appreciate the connection of the various theories it is best to recall the basic or Ising GrossNeveu model which has the Lagrangian, [1],

$$
L^{\mathrm{GN}}=i \bar{\psi}^{i} \not \partial \psi^{i}-m \bar{\psi}^{i} \psi^{i}+g \tilde{\sigma} \bar{\psi}^{i} \psi^{i}-\frac{1}{2} \tilde{\sigma}^{2}
$$

which is renormalizable in two dimensions and $1 \leq i \leq N$. Here the scalar field $\sigma$ is auxiliary and if eliminated produces a theory with a quartic fermion self-interaction. The connection with the Gross-Neveu-Yukawa model is apparent from the Lagrangian, [9],

$$
L^{\mathrm{GNY}}=i \bar{\psi}^{i} \partial \psi^{i}-m \bar{\psi}^{i} \psi^{i}+\frac{1}{2} \partial_{\mu} \tilde{\sigma} \partial^{\mu} \tilde{\sigma}+\frac{1}{2} g_{1} \tilde{\sigma} \bar{\psi}^{i} \psi^{i}+\frac{1}{24} g_{2}^{2} \tilde{\sigma}^{4}
$$

which is renormalizable in four dimensions. In this case the scalar field propagates as a fundamental field and the quartic scalar interaction is necessary to ensure renormalizability. The common 
scalar-fermion interaction shared in both Lagrangians is what underlies the universality and leads to critical point equivalence at the $d$-dimensional Wilson-Fisher fixed point. While (2.2) has now been renormalized in four dimensions to four loops in $[11,12,13]$ the $\varepsilon$ expansion extrapolation of critical exponents to three dimensions has been carried out in [13] using matched Padé approximants. In this a Padé estimate is constructed using data from two and four dimensional perturbation theory. A recent comprehensive analysis collating data from all approaches has been discussed in [15]. To facilitate such a construction the four loop $\beta$-function of (2.1) was provided in [16] as

$$
\begin{aligned}
\beta(g)= & (d-2) g-(N-1) \frac{g^{2}}{\pi}+(N-1) \frac{g^{3}}{2 \pi^{2}}+(N-1)(2 N-7) \frac{g^{4}}{16 \pi^{3}} \\
& +(N-1)\left[-2 N^{2}-19 N+24-6 \zeta_{3}(11 N-17)\right] \frac{g^{5}}{48 \pi^{4}}+O\left(g^{6}\right)
\end{aligned}
$$

where $\zeta_{z}$ is the Riemann zeta function. With this four loop $\beta$-function, estimates of the three exponents were provided in [16] for three dimensions which were commensurate with other techniques.

While this Ising Gross-Neveu universality class has been studied in depth a more apt one for the graphene phase transition of interest is the chiral Heisenberg Gross-Neveu universality class. Its two dimensional Lagrangian is

$$
L^{\mathrm{cHGN}}=i \bar{\psi}^{i} \partial \psi^{i}-m \bar{\psi}^{i} \psi^{i}+g \tilde{\pi}^{a} \bar{\psi}^{i} \sigma^{a} \psi^{i}-\frac{1}{2} \tilde{\pi}^{a} \tilde{\pi}^{a}
$$

where $\sigma^{a}$ are the three Pauli spin matrices. The four dimensional ultraviolet completion is the chiral Heisenberg Gross-Neveu-Yukawa (cHGNY) model which has the Lagrangian

$$
L^{\mathrm{cHGNY}}=i \bar{\psi}^{i} \partial \psi^{i}-m \bar{\psi}^{i} \psi^{i}+\frac{1}{2} \partial_{\mu} \tilde{\pi}^{a} \partial^{\mu} \tilde{\pi}^{a}+\frac{1}{2} g_{1} \tilde{\pi}^{a} \bar{\psi}^{i} \sigma^{a} \psi^{i}+\frac{1}{24} g_{2}^{2}\left(\tilde{\pi}^{a} \tilde{\pi}^{a}\right)^{2}
$$

parallel to (2.2). At present the major activity in evaluating the relevant critical exponents in (2.5) has been with Functional Renormalization Group and perturbative methods, [4, 5, 6, 7, 8]. Given the complementarity of the large $N$ technique with both approaches we note that the $1 / N$ formalism is developed from the underlying universal Lagrangian

$$
L=i \bar{\psi}^{i} \partial \psi^{i}+\pi^{a} \bar{\psi}^{i} \sigma^{a} \psi^{i}+f\left(\pi^{a}\right) .
$$

Here $f\left(\pi^{a}\right)$ represents all additional operators, a small subset of which are relevant to defining a renormalizable Lagrangian in each even critical dimension.

\section{Large $N$ expansion}

We briefly summarize the approach of the critical point large $N$ method developed for scalar models in $[17,18,19]$. The key is that in the approach to criticality the propagators satisfy a power law structure where the exponent is comprised of a canonical and anomalous part. The former is deduced from a dimensional analysis of the $d$-dimensional Lagrangian and the latter reflects the radiative corrections. Both are functions of $d=2 \mu$ with the anomalous part depending on $N$. At criticality $1 / N$ plays the role of the dimensionless coupling constant. Specifically the coordinate space propagators take the scaling forms, [20, 21, 22, 23, 24, 25, 26],

$$
\psi(x) \sim \frac{A \not x}{\left(x^{2}\right)^{\alpha}}\left[1+A^{\prime}\left(x^{2}\right)^{\lambda}\right], \pi(x) \sim \frac{C}{\left(x^{2}\right)^{\gamma}}\left[1+C^{\prime}\left(x^{2}\right)^{\lambda}\right]
$$


where corrections to scaling are included through the exponent $\lambda$. This could be the exponent of the critical $\beta$-function slope for either the chiral Heisenberg Gross-Neveu or chiral Heisenberg Gross-Neveu-Yukawa models. For instance the critical slope of the $\beta$-function of (2.2) was recently determined in [27] to $O(1 / N)$ and to the next order in [28]. The exponents of the fields are given by

$$
\alpha=\mu+\frac{1}{2} \eta, \gamma=1-\eta-\chi_{\pi}
$$

where $\chi_{\pi}$ is the exponent of the universal vertex. For instance, the $1 / N$ expansion of $\eta$ is defined by

$$
\eta(\mu)=\sum_{n=1}^{\infty} \frac{\eta_{n}(\mu)}{N^{n}}
$$

For (2.2) the exponents $\eta, \chi_{\pi}$ and $\lambda=1 /(2 v)$ can be found to $O\left(1 / N^{2}\right)$ in $d$-dimensions by algebraically solving the skeleton Schwinger-Dyson equations when the scaling forms of the propagators are used, [17, 18]. This involves using the so-called master integrals given in [20, 25] but decorating them with the group theory factors derived from the Pauli matrices. It is therefore straightforward to deduce the exponents

$$
\begin{aligned}
& \eta_{1}=-\frac{3 \Gamma(2 \mu-1)}{\mu \Gamma(1-\mu) \Gamma(\mu-1) \Gamma^{2}(\mu)}, \quad \chi_{\pi 1}=-\frac{\mu}{3(\mu-1)} \eta_{1} \\
& \lambda_{1}=-(2 \mu-1) \eta_{1} \quad, \quad \eta_{2}=\left[\frac{(2 \mu-3)}{3(\mu-1)} \Psi(\mu)+\frac{\left(4 \mu^{2}-6 \mu+1\right)}{2 \mu(\mu-1)^{2}}\right] \eta_{1}^{2}
\end{aligned}
$$

where

$$
\Psi(\mu)=\psi(2 \mu-1)-\psi(1)+\psi(2-\mu)-\psi(\mu)
$$

and $\psi(z)$ is the polygamma function. An expression for $v$ at next order requires the evaluation of several four loop graphs with the correction to scaling included in a $\pi^{a}$ line. The result is more involved since

$$
\begin{aligned}
\lambda_{2}= & {\left[\frac{\mu^{2}\left[6 \mu^{2}-3 \mu-8\right]}{6(\mu-1)(\mu-2)} \Theta(\mu)-\frac{2 \mu^{2}(2 \mu-3)}{3(\mu-1)(\mu-2)}\left[\Phi(\mu)+\Psi^{2}(\mu)\right]\right.} \\
& +\frac{2 \mu\left[8 \mu^{2}-16 \mu+7\right]}{3(\mu-1)(\mu-2)^{2} \eta_{1}}+\frac{\left[72 \mu^{8}-604 \mu^{7}+1960 \mu^{6}-3060 \mu^{5}\right]}{18 \mu(\mu-1)^{3}(\mu-2)^{2}} \\
& +\frac{\left[2151 \mu^{4}-146 \mu^{3}-621 \mu^{2}+288 \mu-36\right]}{18 \mu(\mu-1)^{3}(\mu-2)^{2}} \\
& \left.-\frac{(2 \mu-3)\left[18 \mu^{5}-95 \mu^{4}+161 \mu^{3}-86 \mu^{2}-20 \mu+12\right]}{9(\mu-1)^{2}(\mu-2)^{2}} \Psi(\mu)\right] \eta_{1}^{2}
\end{aligned}
$$

with

$$
\Theta(\mu)=\psi^{\prime}(\mu)-\psi^{\prime}(1), \Phi(\mu)=\psi^{\prime}(2 \mu-1)-\psi^{\prime}(2-\mu)-\psi^{\prime}(\mu)+\psi^{\prime}(1) .
$$

Given that the main computational tool to evaluate the large $N$ graphs is the $d$-dimensional conformal integration or uniqueness method of $[16,17]$, but extended to the Gross-Neveu universality class in [20], it is possible to extend $\eta$ to the next order. This requires use of the large $N$ conformal 
bootstrap programme introduced in [18] and then developed for (2.1) in [23, 26, 29]. Application of the method leads to

$$
\begin{aligned}
\eta_{3}=[ & \frac{(2 \mu-3)}{18(\mu-1)^{2}}\left[\Phi(\mu)+3 \Psi^{2}(\mu)\right]-\frac{\left[\mu^{3}+18 \mu^{2}-21 \mu+9\right]}{36(\mu-1)^{2}} \Theta(\mu) \\
& -\frac{\mu^{2}}{3(\mu-1)} \Theta(\mu) \Psi(\mu)-\frac{\mu^{2}}{6(\mu-1)^{3}} \Xi(\mu)-\frac{\mu^{2}}{6(\mu-1)} \Xi(\mu) \Theta(\mu) \\
& -\frac{\left[14 \mu^{7}-15 \mu^{6}-26 \mu^{5}-77 \mu^{4}+324 \mu^{3}-297 \mu^{2}+90 \mu-9\right]}{18 \mu^{2}(\mu-1)^{4}} \\
& \left.-\frac{\left[14 \mu^{5}-37 \mu^{4}-50 \mu^{3}+228 \mu^{2}-183 \mu+27\right]}{18 \mu(\mu-1)^{3}} \Psi(\mu)\right] \eta_{1}^{3}
\end{aligned}
$$

where $\Xi(\mu)$ is related to a two loop self-energy Feynman graph, [18].

\begin{tabular}{|c||l|l|l|l|}
\hline$N=4$ & $1 / v$ & $\eta_{\phi}$ & $\eta$ & $v$ \\
\hline$\varepsilon$ expansion [2,2] [13] & 0.6426 & 0.9985 & 0.1833 & - \\
$\varepsilon$ expansion [3,1] [13] & 0.6447 & 0.9563 & 0.1560 & 1.2352 \\
FRG [30] & 0.795 & 1.032 & 0.071 & 1.26 \\
MC [31] & $(0.98)$ & & $0.20(2)$ & $1.02(1)$ \\
MC [32] & $(1.19)$ & $0.70(15)$ & & $0.84(4)$ \\
Large $N$ & 0.8458 & 1.1849 & 0.1051 & 1.1823 \\
\hline
\end{tabular}

\section{Results}

The main results of [14] were the determination of the three key critical exponents for the chiral Heisenberg Gross-Neveu universality class. A very strong check on their correctness rests in the fact that if one expands each expression in powers of $\varepsilon$ where $d=4-2 \varepsilon$ and compares with the same expansion of the critical exponents derived from the four dimensional renormalization group functions of $[11,12,13]$, then there is exact agreement. One advantage of the large $N$ approach is that three dimensional information can be immediately determined from the $d$-dimensional expressions. In particular we have

$$
\begin{aligned}
\eta & =\frac{4}{\pi^{2} N}+\frac{64}{3 \pi^{4} N^{2}}+\frac{8\left[378 \zeta_{3}-36 \pi^{2} \ln (2)-45 \pi^{2}-332\right]}{9 \pi^{6} N^{3}}+O\left(\frac{1}{N^{4}}\right) \\
\eta_{\phi} & =1+\frac{16\left[3 \pi^{2}+16\right]}{3 \pi^{4} N^{2}}+O\left(\frac{1}{N^{3}}\right) \\
\frac{1}{v} & =1-\frac{16}{\pi^{2} N}+\frac{16\left[144 \pi^{2}+1664\right]}{3 \pi^{4} N^{2}}+O\left(\frac{1}{N^{3}}\right)
\end{aligned}
$$

where there is no $O(1 / N)$ correction for $\eta_{\phi}$ which is related to $\eta+\chi_{\pi}$. As the specific value of $N$ for graphene applications is $N=4$ in our conventions we summarize the current status of exponent estimates in Table 1. Overall the large $N$ and Functional Renormalization Group estimates are broadly similar with $\varepsilon$ summation being less consistent. There have only been a few Monte Carlo 
(MC) evaluations. In comparison with the status of the Ising Gross-Neveu class for the comparable value of $N$ for graphene there is less close agreement between different methods for the chiral Heisenberg Gross-Neveu universality class, [13, 14].

\section{Discussion}

We have summarized the application of the $d$-dimensional critical point large $N$ method to continuum quantum field theories which are believed to underlie a specific phase transition in graphene. For the Ising Gross-Neveu universality class a wide body of methods has produced critical exponent estimates which are commensurate. For the more interesting chiral Heisenberg Gross-Neveu class the picture is currently not as clear. This is in part due to the fact that there has only been activity on this class in more recent years. So the level of precision is not comparable with the Ising class. However the large $N$ exponents are now available to the same order as well as the four dimensional four loop exponents from $[8,13]$ while the FRG results were in effect the first $d$-dimensional exploration of this class. It is therefore clear that now effort should be devoted to bringing estimates from other methods to the same level as the Ising class. Aside from numerical Monte Carlo techniques the determination of the four loop renormalization group functions for the two dimensional chiral Heisenberg Gross-Neveu theory (2.4) would produce matched Padé approximants of the $d$-dimensional behaviour of the exponents.

\section{Acknowledgements}

This work was carried out with the support in part of a DFG Mercator Fellowship. Computations were carried out in part using the symbolic manipulation language FORM, [33, 34].

\section{References}

[1] D. Gross \& A. Neveu, Dynamical symmetry breaking in asymptotically free field theories, Phys. Rev. D10 (1974) 3235.

[2] T.O. Wehling, E. Şaşıoğlu, C. Friedrich, A.I. Lichtenstein, M.I. Katsnelson \& S. Blügel, Strength of effective coulomb interactions in graphene and graphite, Phys. Rev. Lett. 106 (2011) 236805 [arXiv:1101.4007].

[3] M.V. Ulybyshev, P.V. Buividovich, M.I. Katsnelson \& M.I. Polikarpov, Monte-Carlo study of the semimetal-insulator phase transition in monolayer graphene with realistic inter-electron interaction potential, Phys. Rev. Lett. 111 (2013) 056801 [arXiv: 1304 . 3660].

[4] I.F. Herbut, Interactions and phase transitions on graphene's honeycomb lattice, Phys. Rev. Lett. 97 (2006) 146401 [cond-mat/0606195].

[5] I.F. Herbut, V. Juričić \& O. Vafek, Relativistic Mott criticality in graphene, Phys. Rev. B80 (2009) 075432 [arXiv:0904.1019].

[6] S. Sorella, Y. Otsuka \& S. Yunoki, Absence of a spin liquid phase in the Hubbard model on the honeycomb lattice, Sci. Rep. 2 (2012) 992 [arXiv: 1207.1783 ].

[7] F.F. Assaad \& I.F. Herbut, Pinning the order: the nature of quantum criticality in the Hubbard model on honeycomb lattice, Phys. Rev. X3 (2013) 031010 [arXiv: 1304.6340 ]. 
[8] L. Janssen \& I.F. Herbut, Antiferromagnetic critical point on graphene's honeycomb lattice: a functional renormalization group approach, Phys. Rev. B89 (2014) 205403 [arXiv: 1402 . 6277 ].

[9] J. Zinn-Justin, Four fermion interaction near four-dimensions, Nucl. Phys. B367 (1991) 105.

[10] A. Hasenfratz, P. Hasenfratz, K. Jansen, J. Kuti \& Y. Shen, The equivalence of the top quark condensate and the elementary Higgs field, Nucl. Phys. B365 (1991) 79.

[11] L.N. Mihaila, N. Zerf, B. Ihrig, I.F. Herbut \& M.M. Scherer, Gross-Neveu-Yukawa model at three loops and Ising critical behavior of Dirac systems, Phys. Rev. B96 (2017) 165133 [arXiv:1703.08801].

[12] L. Fei, S. Giombi, I.R. Klebanov \& G. Tarnopolsky, Yukawa CFTs and emergent supersymmetry, Prog. Theor. Exp. Phys. (2016) 12C105 [arXiv: 1607.05316$].$

[13] N. Zerf, L.N. Mihaila, P. Marquard, I.F. Herbut \& M.M. Scherer, Four-loop critical exponents for the Gross-Neveu-Yukawa models, Phys. Rev. D96 (2017) 096010 [arXiv: 1709 . 05057].

[14] J.A. Gracey, Large N critical exponents for the chiral Heisenberg Gross-Neveu universality class, Phys. Rev. D97 (2018) 105009 [arXiv: 1801 . 01230].

[15] B. Ihrig, L.N. Mihaila \& M.M. Scherer, Critical behavior of Dirac fermions from perturbative renormalization, arXiv:1806.04977 [cond-mat.str-el].

[16] J.A. Gracey, T. Luthe \& Y. Schröder, Four loop renormalization of the Gross-Neveu model, Phys. Rev. D94 (2016) 125028 [arXiv: 1609 .05071].

[17] A.N. Vasil'ev, Yu.M. Pismak \& J.R. Honkonen, Simple method of calculating the critical indices in the 1/N expansion, Theor. Math. Phys. 46 (1981) 104.

[18] A.N. Vasil'ev, Yu.M. Pismak \& J.R. Honkonen, $1 / N$ expansion: calculation of the exponents $\eta$ and $v$ in the order $1 / N^{2}$ for arbitrary number of dimensions, Theor. Math. Phys. 47 (1981) 465.

[19] A.N. Vasil'ev, Yu.M. Pismak \& J.R. Honkonen, $1 / n$ expansion: calculation of the exponent $\eta$ in the order $1 / n^{3}$ by the conformal bootstrap method, Theor. Math. Phys. 50 (1982) 127.

[20] J.A. Gracey, Calculation of exponent $\eta$ to $O\left(1 / N^{2}\right)$ in the $O(N)$ Gross Neveu model, Int. J. Mod. Phys. A6 (1991) 395, 2755(E).

[21] J.A. Gracey, Anomalous mass dimension at $O\left(1 / N^{2}\right)$ in the $O(N)$ Gross Neveu model, Phys. Lett. B297 (1992) 293.

[22] S.É. Derkachov, N.A. Kivel, A.S. Stepanenko \& A.N. Vasil'ev, On calculation in 1/n expansions of critical exponents in the Gross-Neveu model with the conformal technique, hep-th/9302034.

[23] A.N. Vasil'ev, S.É. Derkachov, N.A. Kivel \& A.S. Stepanenko, The 1/n expansion in the Gross-Neveu model: conformal bootstrap calculation of the index eta in order $1 / n^{3}$, Theor. Math. Phys. 94 (1993) 127.

[24] A.N. Vasil'ev, \& A.S. Stepanenko, The 1/n expansion in the Gross-Neveu model: conformal bootstrap calculation of the exponent $1 / v$ to the order $1 / n^{2}$, Theor. Math. Phys. 97 (1993) 1349.

[25] J.A. Gracey, Computation of $\beta^{\prime}\left(g_{c}\right)$ at $O\left(1 / N^{2}\right)$ in the $O(N)$ Gross Neveu model in arbitrary dimensions, Int. J. Mod. Phys. A9 (1994) 567 [hep-th/9306106].

[26] J.A. Gracey, Computation of critical exponent $\eta$ at $O\left(1 / N^{3}\right)$ in the four fermi model in arbitrary dimensions, Int. J. Mod. Phys. A9 (1994) 727 [hep-th/9306107]. 
[27] J.A. Gracey, Critical exponent $\omega$ in the Gross-Neveu-Yukawa model at $O(1 / N)$, Phys. Rev. D96 (2017) 065015 [arXiv:1707.05275].

[28] A.N. Manashov \& M. Strohmaier, Correction exponents in the Gross-Neveu-Yukawa model at $1 / N^{2}$, Eur. Phys. J. C78 (2018) 454 [arXiv: 1711.02493 ].

[29] J.A. Gracey, The conformal bootstrap equations for the four fermi interaction in arbitrary dimensions, Z. Phys. C59 (1993) 243.

[30] B. Knorr, Critical (chiral) Heisenberg model with the functional renormalisation group, Phys. Rev. B97 (2018) 075129 [arXiv: 1708 . 06200].

[31] Y. Otsuka, S. Yunoki \& S. Sorella, Universal quantum criticality in the metal-insulator transition of two-dimensional interacting Dirac electrons, Phys. Rev. X6 (2016) 011029 [arXiv : 1510.08593 ].

[32] F. Parisen Toldin, M. Hohenadler, F.F. Assaad \& I.F. Herbut, Fermionic quantum criticality in honeycomb and $\pi$-flux Hubbard models: finite-size scaling of renormalization-group-invariant observables from quantum Monte Carlo, Phys. Rev. B91 (2015) 165108 [arXiv: 1411 . 2502].

[33] J.A.M. Vermaseren, New features of FORM, math-ph/0010025.

[34] M. Tentyukov \& J.A.M. Vermaseren, The multithreaded version of FORM, Comput. Phys. Commun. 181 (2010) 1419 [hep-ph/ 0702279$].$ 